\title{
Experimental Investigation of Flood Energy Dissipation through Embankment Followed by Emergent Vegetation
}

\author{
Afzal Ahmed ${ }^{1,2}$, Manousos Valyrakis ${ }^{2 *}$, Abdul Razzaq Ghumman³, Ghufran Ahmed Pasha1, \\ Rashid Farooq ${ }^{4,5}$ \\ ${ }^{1}$ University of Engineering \& Technology Taxila, Rawalpindi 47080, Pakistan \\ ${ }^{2}$ Research Division of Infrastructure and Environment, James Watt School of Engineering, University of Glasgow, United Kingdom \\ ${ }^{3}$ Civil Engineering Department, College of Engineering, Qassim University, Al-Mulida 51431, Saudi Arabia \\ ${ }^{4}$ Department of Civil Engineering, Lakehead University, Thunder Bay, ON P7B 5E1, Canada \\ ${ }^{5}$ Department of Civil Engineering, Faculty of Engineering \& Technology, International Islamic University, Islamabad 44000, Pakistan \\ ${ }^{*}$ Corresponding author, e-mail: Manousos.Valyrakis@glasgow.ac.uk
}

Received: 21 March 2021, Accepted: 16 July 2021, Published online: 16 August 2021

\begin{abstract}
The combination of hard (artificial) and soft (natural) solutions i.e., composite defense systems against flooding and tsunami opens a new window for engineering innovation for researchers nowadays. In this study, the experimental investigation of flood energy dissipation phenomena through composite defense systems comprising of embankment and rigid vegetation models in an open channel flume, is conducted. The flow regime through the composite defense system is classified in two main types, which are further subdivided in two sub-categories. Various combinations of embankment and vegetation and spacing between embankment and vegetation are analyzed. Against the selected range of initial Froude numbers, three different sizes of embankment models, three spacings between the embankment and vegetation $\left(L_{d v}\right)$ and vegetated corridors of two different porosities $\left(P_{R}\right)$, are tested to examine the effect of these three parameters on the characteristics of the generated hydraulic jumps and flood energy dissipation within the defense system. It is found that embankment size and vegetation porosity have a greater impact on flood energy dissipation while the selected range of $L_{d v}$ is less effective. Amongst the assessed composite flood defense systems, the maximum energy dissipation (55\%) is observed for the combination of maximum embankment height and vegetation porosity (93\%). For fixed combinations of embankment size and $L_{d v^{\prime}}$ the maximum increase of energy dissipation (18\%) is found for decreasing vegetation porosity from $97 \%$ to $93 \%$.
\end{abstract}

\section{Keywords}

flood, energy, hydraulic jump, embankment, vegetation, porosity

\section{Introduction}

The deadly effects of natural disasters like floods and tsunamis have been increasing since the last decade. To cope with these damages the researchers are now focusing on composite defense structures [1]. In this regard the composite defense structures in the form of hard and soft solutions are introduced in the field of experimental hydraulics and water resources. In response to serious damage due to Great East Japan tsunami in 2011, several authors have studied composite defense structure comprising of coastal forests with dikes with or without moat/depression [2, 3]. Various other studies have been conducted to analyze the effectiveness of composite defense structures against flooding. For example, such experimental studies have been conducted to quantify the reduction of velocity and fluid force of a tsunami wave behind a double layered vegetation [3, 4], and to analyze in detail the energy dissipation through composite defense systems comprising of vegetation and backward facing steps $[5,6]$. Moreover, the combined effects of sea embankments and coastal forests has also been tested [7] against moats with and without vegetation [8] and a double embankment system has been studied experimentally by varying the spacing between the embankments to understand the energy dissipation through it [9]. Many other studies of composite defense structures [10,11] including the analysis of embankment and vegetation with moat [12], embankment upstream barriers [13] have been conducted, in the recent past. It has been reported in the literature that the flow within such composite defense systems 
becomes more complex due to the formation of hydraulic jump [14, 15]. Previous experimental investigations have focused on obtaining a better understanding of the energy dissipation through such composite (embankment and vegetation) flood defense systems and categorizing the formation of hydraulic jump within these into various types, depending upon the location of the formation of hydraulic jump [2]. Optimal arrangements of composite defense system consisting of dikes, moats, and vegetation zones, has been analyzed under unsteady flow conditions [16]. A series of experiments was also conducted in the past to investigate energy dissipation through a composite defense system comprising of an embankment, moats of various shapes and vegetation under steady flow conditions. The hydraulic jump was also categorized, and the results were compared with single defense systems of only vegetation of varying densities [8]. However, the experimental investigation of optimal arrangements of composite defense systems comprising of dikes and emergent vegetation to dissipate flood energy is still required to be further investigate [6], towards increasing the efficiency and robustness of flood defense systems, especially for places under increased urbanization pressure where space is limited.

The aim of this study is to introduce a composite defense system that dissipates flood energy within the selected ranges of the embankment size, spacings between the embankment and vegetation $\left(L_{d v}\right)$ and vegetated corridors of two different porosities $\left(P_{R}\right)$. The flood energy dissipation phenomena are analyzed experimentally by providing two energy dissipating structures in the sequence of embankment on upstream and emergent vegetation on the downstream side. The effect of various parameters including the dimensions of embankment model, spacing between dike and vegetation and the density (in the form of porosity) of vegetation on energy dissipation are tested and the best solution is recommended, which is not reported previously. The results from this study are not location specific and can be generalized to hold for any real-world scenario under proper geometric and dynamic scaling. However, for this study parameters ranges such as for the flow conditions, vegetation densities and dike dimensions, are selected using historical data from Indus River, in Pakistan.

\section{Materials and methods}

\subsection{Flume characteristics and flow conditions}

The Fig. 1(a) shows the experimental setup of a composite defense system in the sequence of embankment and vegetation in a glass sided open channel rectangular flume $(10 \mathrm{~m} \times 0.31 \mathrm{~m} \times 0.50 \mathrm{~m})$ with a constant bed slope of $1 / 1000$. Froude similarity is used to set the model scale of the laboratory experiments as it has been also used in a previous study [17]. All the experiments with composite defense systems discussed in the current paper are performed under steady, subcritical flow conditions $\left(F r_{o}=V /(g h) 0.5<1\right.$, where $F r_{o}=$ initial Froude number, $V=$ depth-averaged velocity, $g$ is gravitational acceleration $\left(\mathrm{m} / \mathrm{s}^{2}\right)$, and $h$ is water depth $(\mathrm{m})$ as the historical field data of Indus River has shown that the Froude number attained in the river is less than unity [8]. Hence, the selected range of Froude numbers for the current study is subcritical i.e., in the range of $0.40-0.65$. In the current study, the scale of 1:100 is kept for all cases. Seven values of initial Froude number $\left(F r_{o}\right)$ are $0.40,0.44,0.50,0.57,0.60,0.63$ and 0.65 are selected, while the corresponding flow depths are $0.045 \mathrm{~m}, 0.053 \mathrm{~m}, 0.068 \mathrm{~m}, 0.071 \mathrm{~m}, 0.077 \mathrm{~m}, 0.081 \mathrm{~m}$ and $0.085 \mathrm{~m}$ respectively. The initial Froude numbers $\left(F r_{o}\right)$ are calculated by measuring the flow depth and the corresponding velocities in the flume without placing any structure in the channel. The water surface profiles are drawn by measuring the depth of flow $(h)$ within the composite defense system at an interval of $1-5 \mathrm{~cm}$ depending upon the fluctuations and undulations. The rail mounted moveable point gauge of sub-millimeter accuracy is used for measuring the water depth. The flow meter provides the reading of flow in the channel and the depth average velocity $(V)$ is calculated by the relation $Q=A V$ (where, $Q$ is discharge, $\mathrm{A}$ is cross-sectional area and $\mathrm{V}$ is the mean velocity) same as used previously $[7,16]]$.

In this study, a composite flood defense system in the form of dike and riverine vegetation is modelled in a laboratory flume and energy dissipation is analyzed in detail and water surface profiles are categorized considering the characteristics of hydraulic jumps. Combinations of three different dimensions of embankment, three different spacings between the toe of embankment and the upstream edge of vegetation ( $L_{d v}=10 \mathrm{~cm}, 15 \mathrm{~cm}$ and $20 \mathrm{~cm}$ ) and two different porosities of vegetation ( $97 \%$ and $93 \%$ ) are tested to assess flood energy dissipation and hydraulic jump formation. Hence, various combinations of embankment size, vegetation zone porosities and their distance $\left(L_{d v}\right)$, are tested as shown in Table 1 (with initial Froude numbers ranging from 0.40 to 0.65 ). The specific energy is defined as the summation of potential head $(h)$ and the velocity head $\left(V^{2} / 2 g\right)[14,18,19]$. 
Table 1 Experimental conditions of composite defense system

\begin{tabular}{|c|c|c|c|c|c|c|c|c|}
\hline Case ID & Froude No. & $\begin{array}{c}\text { Discharge } \\
(\text { lit/s) }\end{array}$ & $\begin{array}{l}\text { PR } \\
(\%)\end{array}$ & $\begin{array}{c}\text { Type of } \\
\text { Dike }\end{array}$ & $\begin{array}{c}d \\
(\mathrm{~cm})\end{array}$ & $\begin{array}{c}J \\
(\mathrm{~cm})\end{array}$ & $\begin{array}{c}L_{d v} \\
(\mathrm{~cm})\end{array}$ & $\begin{array}{c}W_{v} \\
(\mathrm{~cm})\end{array}$ \\
\hline ES10V97 & $0.40,0.44,0.50,0.57,0.60,0.63,0.65$ & $3,4.3,7.0,8.75,10.3,11.8,12.9$ & 93 & Small & 0.3 & 1.88 & 10 & 18.3 \\
\hline ES15V97 & $0.40,0.44,0.50,0.57,0.60,0.63,0.65$ & $3,4.3,7.0,8.75,10.3,11.8,12.9$ & 93 & Small & 0.3 & 1.88 & 15 & 18.3 \\
\hline ES20V97 & $0.40,0.44,0.50,0.57,0.60,0.63,0.65$ & $3,4.3,7.0,8.75,10.3,11.8,12.9$ & 93 & Small & 0.3 & 1.88 & 20 & 18.3 \\
\hline EM10V97 & $0.40,0.44,0.50,0.57,0.60,0.63,0.65$ & $3,4.3,7.0,8.75,10.3,11.8,12.9$ & 93 & Medium & 0.3 & 1.88 & 10 & 18.3 \\
\hline EM15V97 & $0.40,0.44,0.50,0.57,0.60,0.63,0.65$ & $3,4.3,7.0,8.75,10.3,11.8,12.9$ & 93 & Medium & 0.3 & 1.88 & 15 & 18.3 \\
\hline EM20V97 & $0.40,0.44,0.50,0.57,0.60,0.63,0.65$ & $3,4.3,7.0,8.75,10.3,11.8,12.9$ & 93 & Medium & 0.3 & 1.88 & 20 & 18.3 \\
\hline EL10V97 & $0.40,0.44,0.50,0.57,0.60,0.63,0.65$ & $3,4.3,7.0,8.75,10.3,11.8,12.9$ & 93 & Large & 0.3 & 1.88 & 10 & 18.3 \\
\hline EL15V97 & $0.40,0.44,0.50,0.57,0.60,0.63,0.65$ & $3,4.3,7.0,8.75,10.3,11.8,12.9$ & 93 & Large & 0.3 & 1.88 & 15 & 18.3 \\
\hline EL20V97 & $0.40,0.44,0.50,0.57,0.60,0.63,0.65$ & $3,4.3,7.0,8.75,10.3,11.8,12.9$ & 93 & Large & 0.3 & 1.88 & 20 & 18.3 \\
\hline ES10V93 & $0.40,0.44,0.50,0.57,0.60,0.63,0.65$ & $3,4.3,7.0,8.75,10.3,11.8,12.9$ & 97 & Small & 0.3 & 1.254 & 10 & 8.2 \\
\hline ES15V93 & $0.40,0.44,0.50,0.57,0.60,0.63,0.65$ & $3,4.3,7.0,8.75,10.3,11.8,12.9$ & 97 & Small & 0.3 & 1.254 & 15 & 8.2 \\
\hline ES20V93 & $0.40,0.44,0.50,0.57,0.60,0.63,0.65$ & $3,4.3,7.0,8.75,10.3,11.8,12.9$ & 97 & Small & 0.3 & 1.254 & 20 & 8.2 \\
\hline EM10V93 & $0.40,0.44,0.50,0.57,0.60,0.63,0.65$ & $3,4.3,7.0,8.75,10.3,11.8,12.9$ & 97 & Medium & 0.3 & 1.254 & 10 & 8.2 \\
\hline EM15V93 & $0.40,0.44,0.50,0.57,0.60,0.63,0.65$ & $3,4.3,7.0,8.75,10.3,11.8,12.9$ & 97 & Medium & 0.3 & 1.254 & 15 & 8.2 \\
\hline EM20V93 & $0.40,0.44,0.50,0.57,0.60,0.63,0.65$ & $3,4.3,7.0,8.75,10.3,11.8,12.9$ & 97 & Medium & 0.3 & 1.254 & 20 & 8.2 \\
\hline EL10V93 & $0.40,0.44,0.50,0.57,0.60,0.63,0.65$ & $3,4.3,7.0,8.75,10.3,11.8,12.9$ & 97 & Large & 0.3 & 1.254 & 10 & 8.2 \\
\hline EL15V93 & $0.40,0.44,0.50,0.57,0.60,0.63,0.65$ & $3,4.3,7.0,8.75,10.3,11.8,12.9$ & 97 & Large & 0.3 & 1.254 & 15 & 8.2 \\
\hline EL20V93 & $0.40,0.44,0.50,0.57,0.60,0.63,0.65$ & $3,4.3,7.0,8.75,10.3,11.8,12.9$ & 97 & Large & 0.3 & 1.254 & 20 & 8.2 \\
\hline
\end{tabular}

Note: The case ID consists of three parts i.e., the type of embankment, spacing between embankment and vegetation model and the porosity of vegetation. For example, ID ES10V97 represents ES = small embankment, $10=$ spacing between embankment and vegetation and V97 = vegetation having porosity $97 \%$.

\subsection{Embankment and vegetation conditions}

The recommended height of embankment (dike) on Indus River in Pakistan is in the range of about 4-7 $\mathrm{m}$ high in addition to $1.2-1.8 \mathrm{~m}$ freeboard [20]. In the current study, three different embankment sizes as part of composite defense structures are tested to assess their efficiency at dissipating flood energy. The selected height of embankments (including the freeboard) is $4 \mathrm{~m}, 5.5 \mathrm{~m}$ and $7 \mathrm{~m}$ respectively. Therefore, for the physical modelling, under a geometric scaling of 1:100, the height of the embankment model is kept at $4 \mathrm{~cm}, 5.5 \mathrm{~cm}$ and $7 \mathrm{~cm}$, respectively, as shown in Fig. 1(b). To ensure geometric similarity, trapezoidal embankment models made of wood are used for the current laboratory experiments.

During a high stage flood, water overtops the embankment and may be routed through the floodplain. The direction of flow towards the weir-like obstacles/embankments in the floodplains can take place at various orientations i.e., at an oblique or perpendicular angle $[18,19]$. In the current study the effect of flow direction relative to the flood defenses is not being assessed, thus perpendicular flow conditions are considered, consistent to past research $[9,22,23]$.
For modelling of vegetation, the Eucalyptus species are selected. The literature mentions that the average trunk diameter $(d)$ of Eucalyptus tree is in the range of 0.11-0.33 $\mathrm{m}$ and the tree crown height of Eucalyptus tree is greater than the flood stage in Pakistan [23]. Keeping in views the above limitations the diameter of vegetation is set as $0.003 \mathrm{~m}$ due to the scale of 1:100. To ensure geometrical similarity, vegetation elements made of steel rods (as also used previously $[9,24,25]$ are vertically inserted on the bed of the flume, as shown in Fig. 1(a). The effect of any floodplain slope is considered to be negligible compared to that of the river banks and defense works. The density of the vegetation can be represented by finding the porosity of vegetation by Eq. (1).

$P_{R}=1-n_{t} \pi d^{2} / 4$,

where, $P_{R}=$ porosity $(\%), n_{t}=$ number of vegetation elements per unit area and $d=$ diameter of vegetation. In this study two different porosities of vegetation (93\% and 97\%) are used. The embankment model placed at a spacing of $400 \mathrm{~cm}$ from the channel inlet and the vegetation model (having staggered arrangement) is placed on the downstream side of embankment model, as shown in Fig. 1(c). 


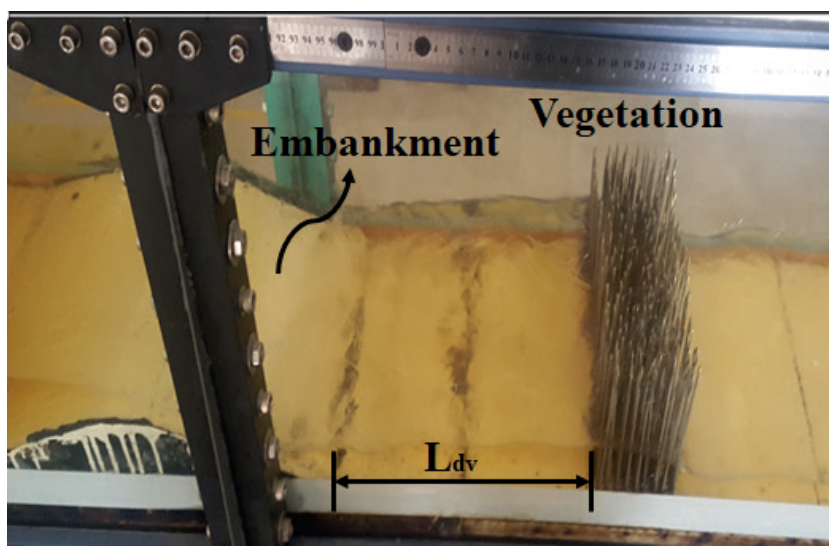

(a)

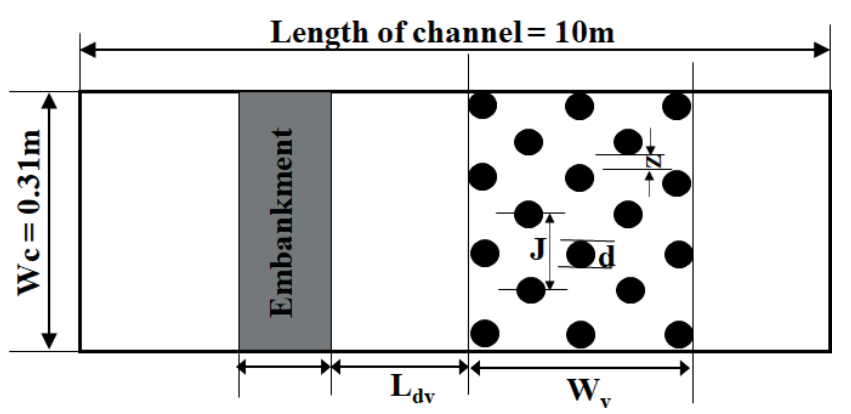

(b)
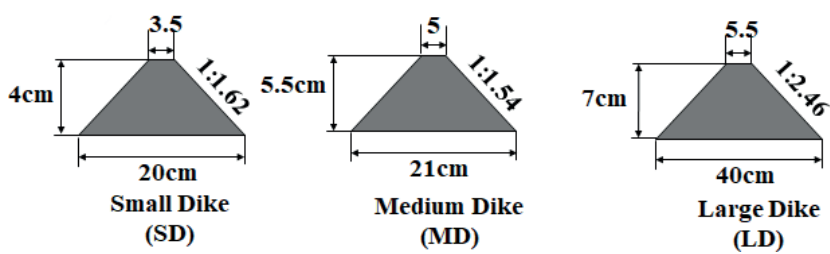

(c)

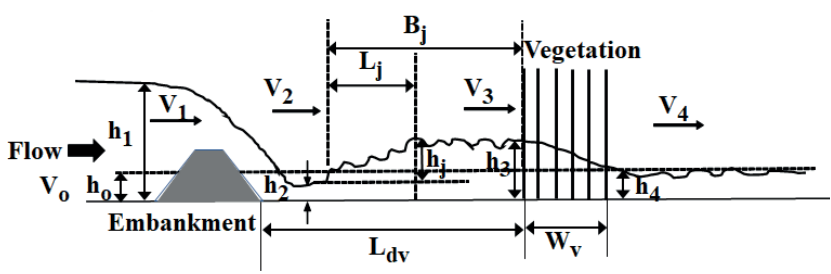

(d)

Fig. 1 Experimental setup; (a) Experimental flume, (b) embankment models of different sizes, (c) Top view of experimental setup and (d) Flow structure scheme

\subsection{Non-dimensional parameters}

The main Fig. 1(d) shows the schematic view of flow regime in the composite defense system representing various dominant parameters including the width of vegetation $\left(W_{v}\right)$, distance between the downstream toe of embankment and the upstream edge of vegetation model $\left(L_{d v}\right)$, the gravitational acceleration $g\left(\mathrm{~m} / \mathrm{s}^{2}\right)$ and the hydraulic jump parameters $\left(L_{d v}, J, d, h_{0}, V_{0}, h_{1}, V_{1}, h_{2}, V_{2}, h_{3}, V_{3}, h_{4}\right.$,
$\left.V_{4}, B_{j}, L_{j}, h_{j}, E_{1}, E_{2}\right)$, where, $L_{d v}=$ distance between the embankment and vegetation model, $J=$ distance between cylinders in the cross stream direction, $d=$ diameter of cylinder simulating vegetation elements, $h_{0}=$ normal depth without any model, $V_{0}=$ initial velocity, $h_{1}=$ water depth upstream of the embankment, $V_{1}=$ velocity upstream of the embankment, $h_{2}=$ water depth at the location of hydraulic jump formation, $V_{2}=$ velocity at the location of hydraulic jump formation, $h_{3}=$ water depth upstream the vegetation zone, $V_{3}=$ velocity upstream the vegetation zone, $h_{4}=$ water depth downstream the vegetation zone, $V_{4}=$ velocity downstream the vegetation zone, $B_{j}=$ the distance of the toe of the hydraulic jump to the upstream face of vegetation zone; $L_{j}=$ the horizontal distance from the start of the jump to the location where the surface roller ends, $h_{j}=$ height of hydraulic jump, $E_{1}=$ specific energy on the upstream of embankment and $E_{2}=$ specific energy on the downstream of vegetation. According to Buckingham $\pi$ theorem these parameters could be coupled into independent dimensional parameter. By using the Buckingham $\pi$ theorem, the function $\left(f_{1}\right)$ can be represented as a function of the following independent dimensionless parameters.

$f\left(\begin{array}{l}F r_{0}, P r, F r_{1}, F r_{2}, F r_{3}, F r_{4}, P^{\prime}, L_{j} / h_{1}, \\ h_{j} / h_{3}, h_{2} / h_{1}, h_{3} / h_{1}, \Delta E / E_{1}\end{array}\right)=0$,

where $P_{r}$ (porosity) $=1-n \pi d^{2} / 4$, where $n=2 / \sqrt{ } 3 d^{2}$ is the number of trees per unit area, $F r_{o}=V_{0} /\left(g h_{0}\right)^{1 / 2}$ is the initial Froude number without any model in the channel, $F r_{1}=V_{1} /\left(g h_{1}\right)^{1 / 2}$ is the Froude number upstream the model embankment, $F r_{2}=V_{2} /\left(g h_{2}\right)^{1 / 2}$ is the Froude number downstream the model embankment, $F r_{3}=V_{3} /\left(g h_{3}\right)^{1 / 2}$ is the Froude number upstream the vegetation zone and $F r_{4}=V_{4} /\left(g h_{4}\right)^{1 / 2}$ is the Froude number downstream the vegetation zone, $P^{\prime}\left(=B_{j} / L_{d v}\right)$ is the nondimensional position of the start of the hydraulic jump, $\Delta E=E_{2}-E_{1}$ is the energy loss through the composite defense system and $\Delta E / E_{1}$ is the relative energy loss through the composite defense system. The selected spacing between the vegetation cylinders $(J)$ are $1.88 \mathrm{~cm}$ and $1.25 \mathrm{~cm}$ and corresponding width of vegetation $\left(W_{v}\right)$ is $18.36 \mathrm{~cm}$ and $8.20 \mathrm{~cm}$ for sparse and intermediate vegetation porosities, respectively, same as used previously [8].

\subsection{Hydraulic jump formation}

Generally, in an open channel flow, when the flow changes its state from supercritical to subcritical, a hydraulic jump is formed [26]. It is important to control the position of 
a hydraulic jump to control erosion downstream and ensure the stability of the hydraulic structure as well as reduce downstream flow energy. Thus, the jump type and its classification in the hydraulic structures is essential for understanding the mitigation effect of a defense system [2]. In this research, the formation of hydraulic jump is categorized depending upon the region of its formation. In type A-jump, hydraulic jump is formed in between the embankment and vegetation i.e., Ldv, whereas type B-jump is formed on the downstream glacis of the embankment. Fig. 2(a-b) represents the flow regime of type A and type B hydraulic jump and associated parameters. Both hydraulic jump types are further classified into two subcategories i.e., type A-1, A-2 and B-1, B-2. Where, A-1 and B-1 are the types of jumps in which the water depth inside vegetation patch is higher than the depth on upstream and downstream side of vegetation and A-2 and B-2 are the jumps in which the upstream water depth is higher than the depth inside and downstream of vegetation. The hydraulic jump classification is presented in Fig. 3(a-d).

\subsection{Energy dissipation}

Energy dissipation $\left(\Delta E=E_{1}-E_{2} / E_{1}\right)$ caused by the composite defense system is calculated as the difference between the energy head $\left(E_{1}\right)$ on the upstream side of embankment and energy head $\left(E_{2}\right)$ on the downstream side of vegetation. In this study, three main factors including the type of embankment, distance between the embankment and vegetation and the porosity/density of vegetation are analyzed in detail. To examine the effect of these parameters on energy dissipation, each is analyzed by keeping the other two factors constant. To examine the difference in mean values of energy dissipation of all the cases, the least significant difference $(L S)$ method is applied as a pairwise comparison between the mean values of different cases as reported previously [27]. In this method the least significant difference between two means is calculated by the following formula, $L S_{c r}=t\left(M S W \times\left(1 / N_{1}+1 / N_{2}\right)^{1 / 2}\right.$, where, $L S_{c r}=$ critical least significant difference, $t=$ critical value for $\alpha=0.05$, $\mathrm{MSW}=$ mean square within, obtained from ANOVA test, $N_{1}$ and $N_{2}=$ number of values used to calculate means (i.e., $\mu_{1}$ and $\mu_{2}$ ) of pairs. If the difference between the means of a pair is higher than the critical $L S_{c r}$ value means there is a significant difference (SD) between the two means otherwise no significant difference (NSD) occurs between the two means [27].

\section{Results}

\subsection{Classification of flow regimes}

In the current study, the hydraulic jump is formed either on the bed of the channel in between the embankment and the vegetation or on the downstream glacis of the embankment. Fig. 3(a-d) shows the classification of hydraulic jumps. Water surface profiles are sketched for all the cases. The four sketches representing the various types of hydraulic jumps are shown in Fig. 4(a-d). The experimental views presenting all types of hydraulic jumps are shown in Fig. 5(a-d).

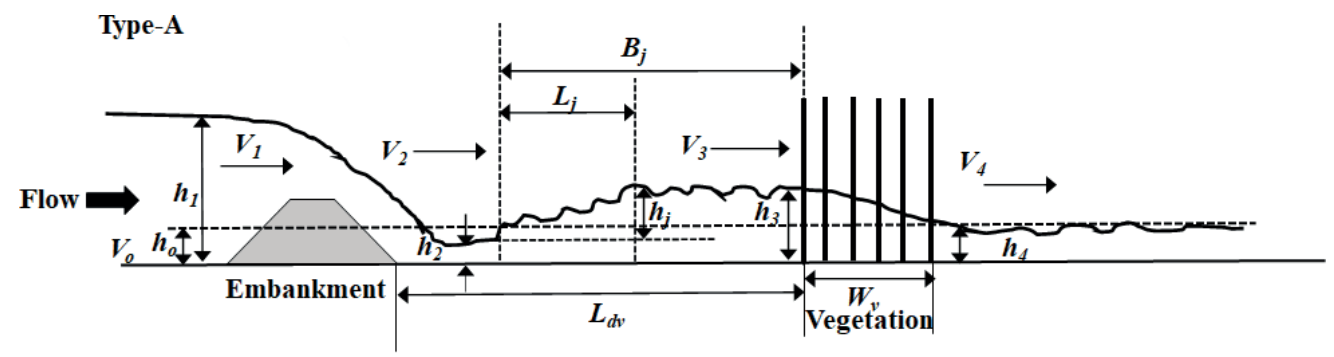

(a)

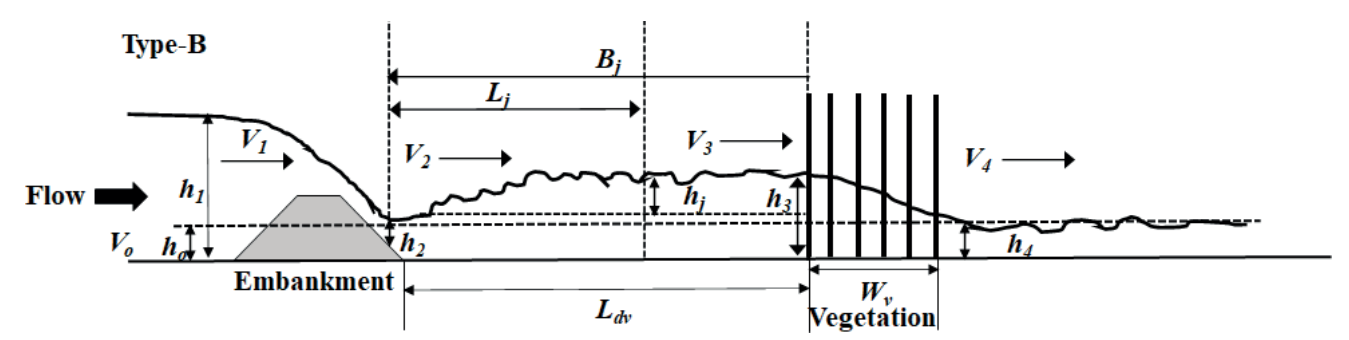

(b)

Fig. 2 Sketch of the water surface profiles and hydraulic jump type with the definition of hydraulic jump parameters: (a) Jump occurs in between the embankement and vegetation models (Type A) and (b) jump occurs on the downstream glacis of the embankement model (Type-B) 


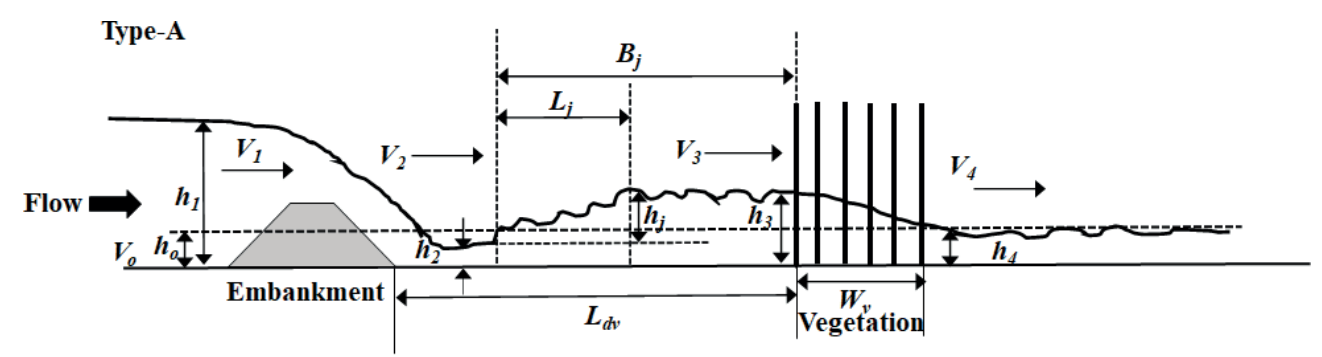

(a)

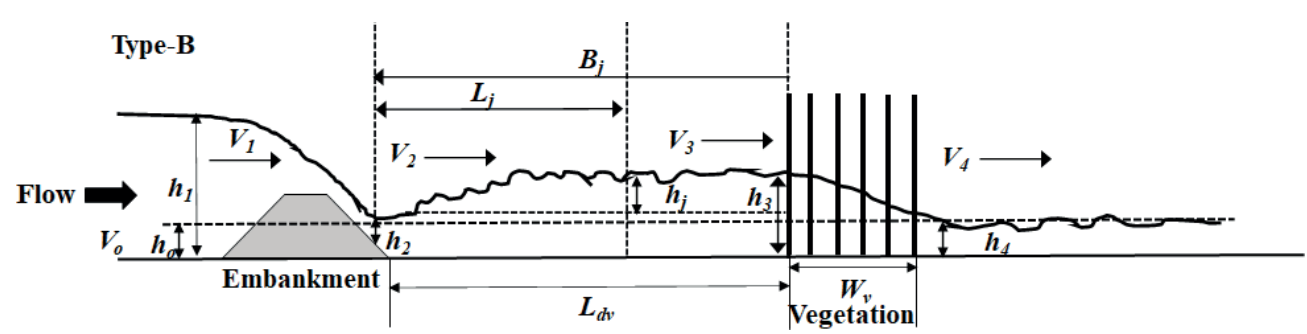

(b)

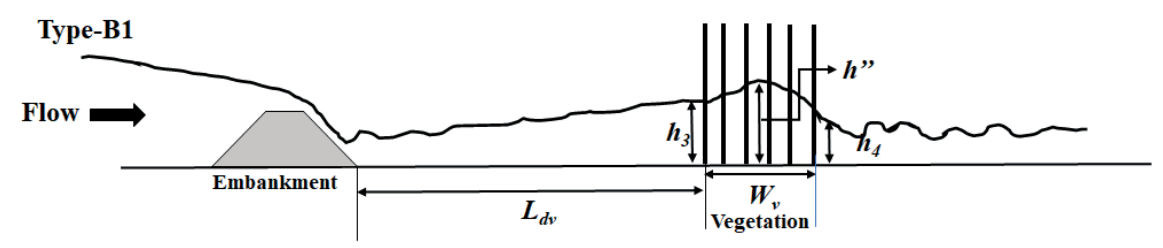

(c)

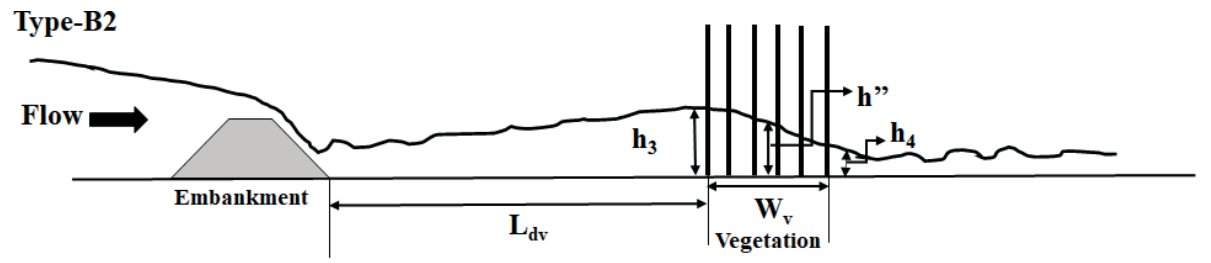

(d)

Fig. 3 Sketch to hydraulic jump classification observed in the current study, (a) Type-A1, (b) Type-A2, (c) Type-B1 and (d) Type B-2

The results show that for small embankment cases with 97\% porosity, for ES10V97, type A2 is observed against $F r_{o}=0.40$ and 0.44 , while type B1 is observed for the remaining higher values of $F r_{o}=0.50-0.65$. For ES15V97, A2 hydraulic jump is observed against $F r_{o}=0.40,0.44$ and 0.50 while B1 hydraulic jump is observed against the remaining values of $F r_{o}=0.47-0.65$. For ES20V97, only A1 type hydraulic jump is observed. For all the cases of small embankment with 93\% porosity, only A2 type jumps are observed against each $F r_{o}$. The quantitative analysis shows that for all the cases of small embankment with $97 \%$ and $93 \%$ porosity, $16.7 \%$ cases show A1 type hydraulic jump, $62 \%$ A2 and $21.4 \%$ B1 type is observed.

For medium embankment cases with 97\% porosity, for EM10V97 and EM15V97, type A1 is observed against $F r_{o}=0.40$ and 0.44 and type B1 is observed against $F r_{o}=0.50-0.63$ and type B2 is observed for $F r_{o}=0.65$. For EM20V97, B1 type hydraulic jump is observed against $F r_{o}=0.40-0.57$ and type B2 is observed against $F r_{o}=0.60-0.65$. The quantitative analysis shows that for all the cases of medium embankment with $97 \%$ and $93 \%$ porosity, 9.5\% cases show A1 type hydraulic jump, 50\% A2, 28.5\% B1 and 12\% B2 type is observed. For large embankment cases with 97\% porosity, for EL10V97, only B1 type hydraulic jump is observed against all the values of selected range of $F r_{o}=0.40-0.65$. For EL15V97, type B1 is observed against $F r_{o}=0.40$ and 0.44 and type B2 is observed against $F r_{o}=0.50-0.65$. For EL20V97, B1 type hydraulic jump is observed against $F r_{o}=0.40-0.50$ and type B2 is observed against $F r_{o}=0.57-0.65$. The quantitative analysis shows that for all the cases of large embankment with $97 \%$ and $93 \%$ porosity, $35.7 \%$ cases show B1 type hydraulic jump and $64.3 \%$ cases show B2 type. The hydraulic jump of type A1, B1, A2 and B2 are indicated in Fig. 4(a-d), respectively and the experimental views of the relavent hydraulic jump classifications are shown in Fig. 5(a-b). 
(a)

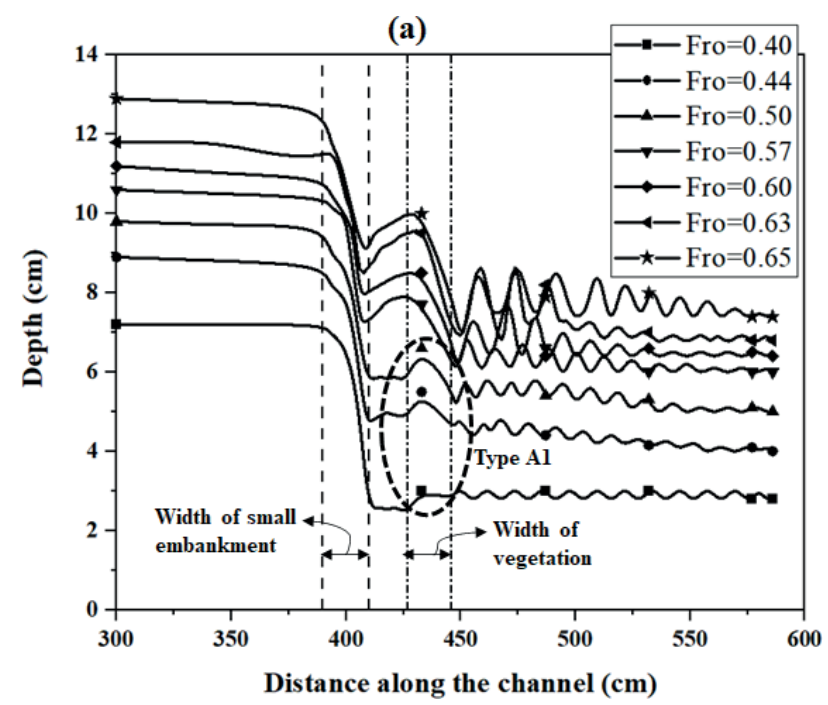

(c)

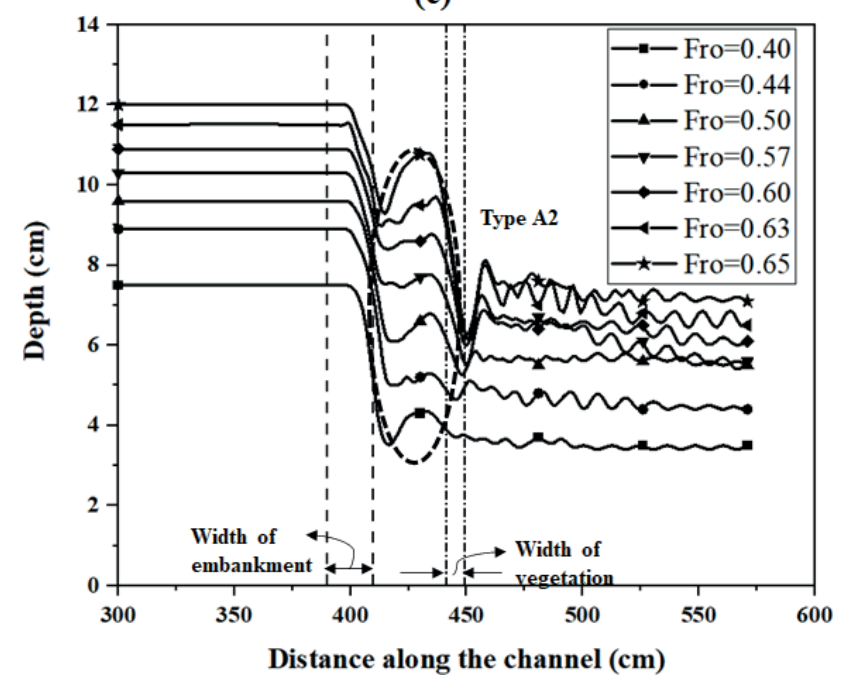

(b)

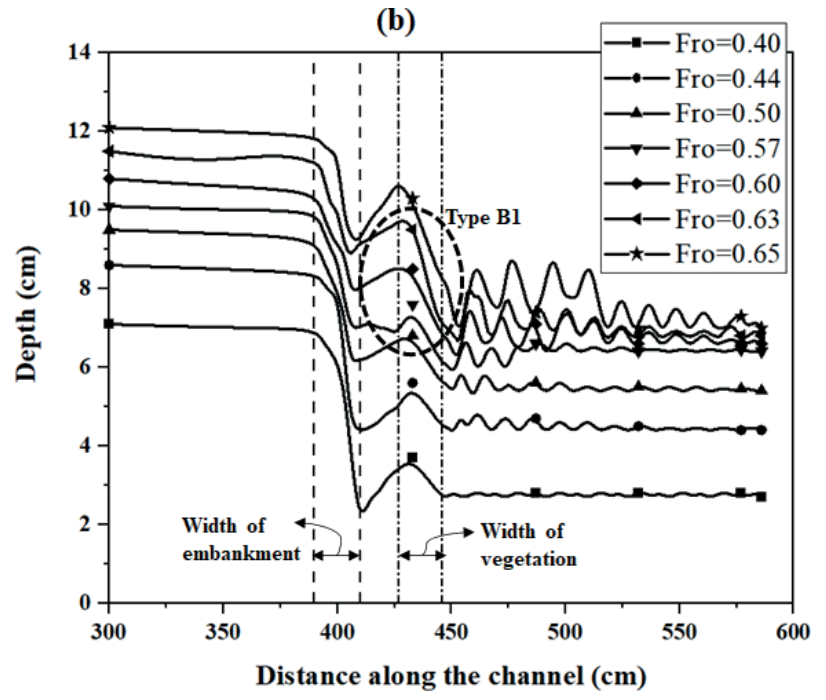

(d)

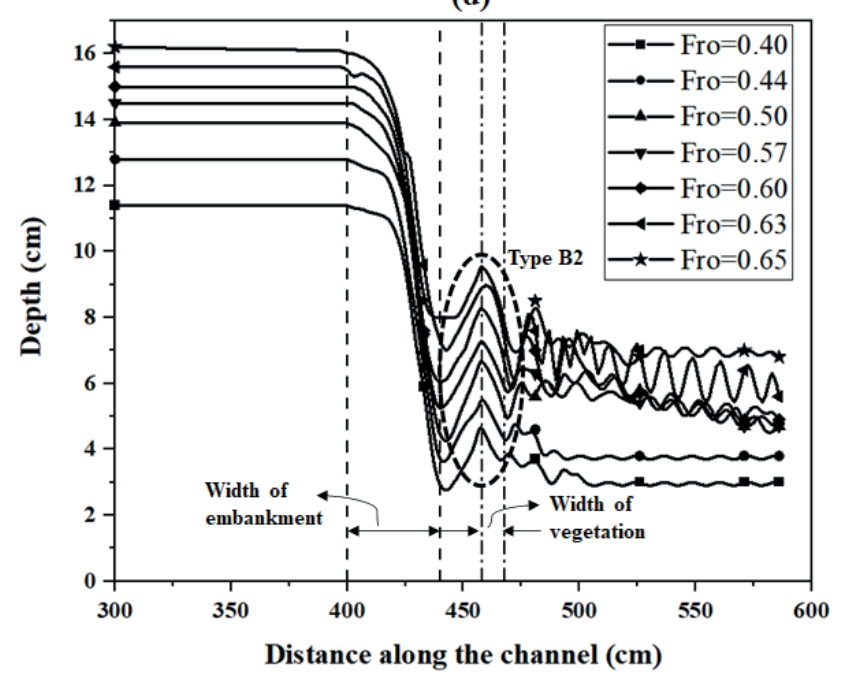

Fig. 4 Water surface profiles (a) ES15V97 (type A1), (b) EM15V97 (type B1), (c) EM15V93 (type A2) and (d) EL15V93 (type B)

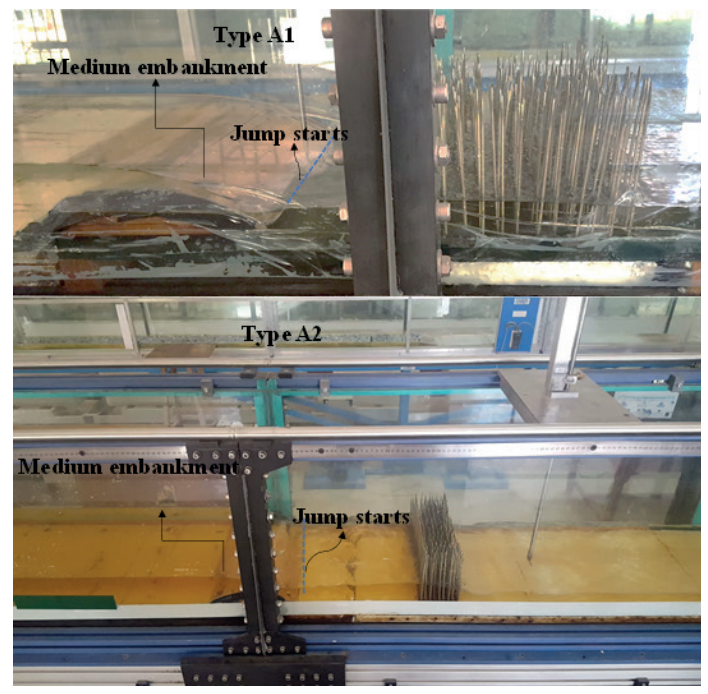

(a)

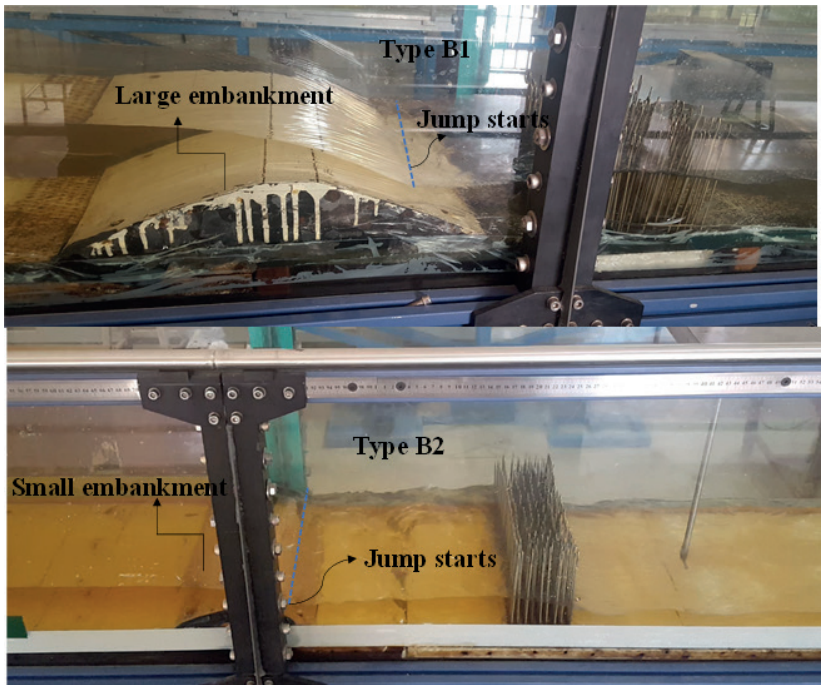

(b)

Fig. 5 Hydraulic jump classification (a) Type A-1 A-2, (b) Type B-1 and B-2 


\subsection{Relative energy loss (\%)}

3.2.1 Constant distance $\left(L_{d v}\right)$ and porosity $\left(P_{R}\right)$ with varying type of embankment

To examine the effect of embankment size, the relative energy loss (\%) is calculated by keeping $L_{d v}$ and $P_{R}$ constant and assessing three embankment sizes (small, medium and large). The analysis has shown that relative energy dissipation is maximum for the embankment with the greatest height (large embankment), resulting in higher difference between $E_{1}$ and $E_{2}$. The maximum average energy loss is $55 \%$ in EL15V93 case, and the energy loss decreases by increasing the initial Froude number $\left(\mathrm{Fr}_{o}\right)$ for all the cases. By increasing the size of embankment from

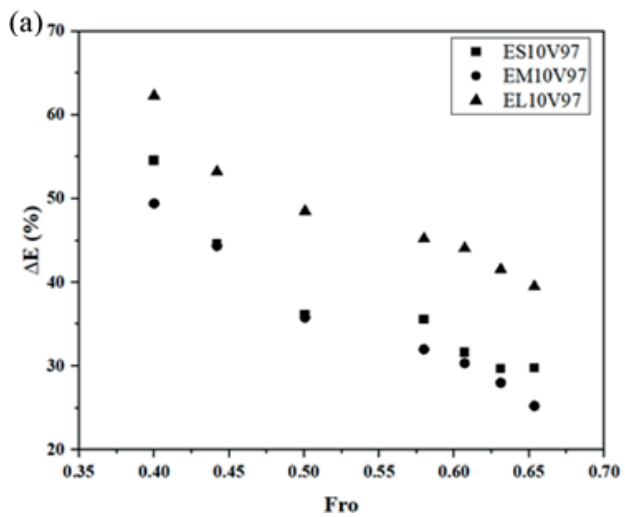

(c)

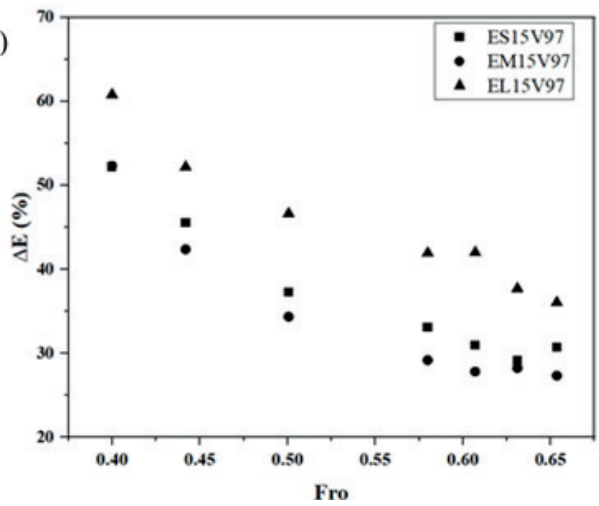

(e)

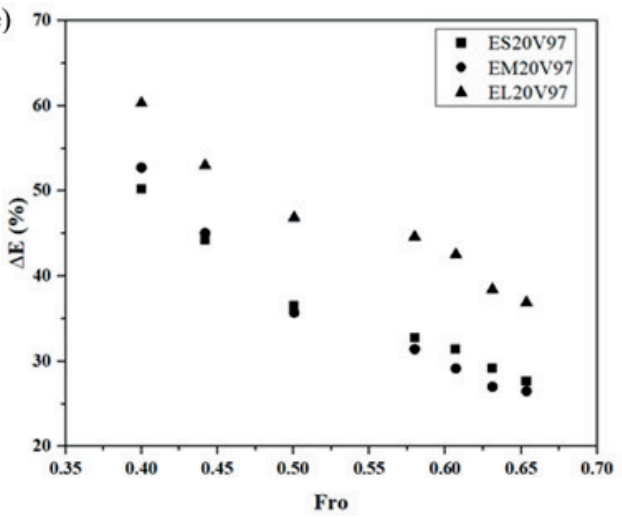

small to medium, the variation in energy dissipation is not prominent, however, a considerable increment (approximately $18 \%$ ) in energy dissipation is observed when the embankment type is changed from medium to large. The energy dissipation decreases by increasing $F r_{o}$ as shown in Fig. 6(a-f). The calculations of least square difference method also showed that the mean difference $\left(\mu_{1}-\mu_{2}\right)$ of small and medium embankment cases are less than LScr, which means that there is no significant difference (NSD) in the two cases as mentioned in Table 2 (Appendix A). However, the mean difference for the pairs of small to large and medium to large embankment, is higher than $L S_{c r}$, which means that a significant difference (SD) is resulted.

(b)

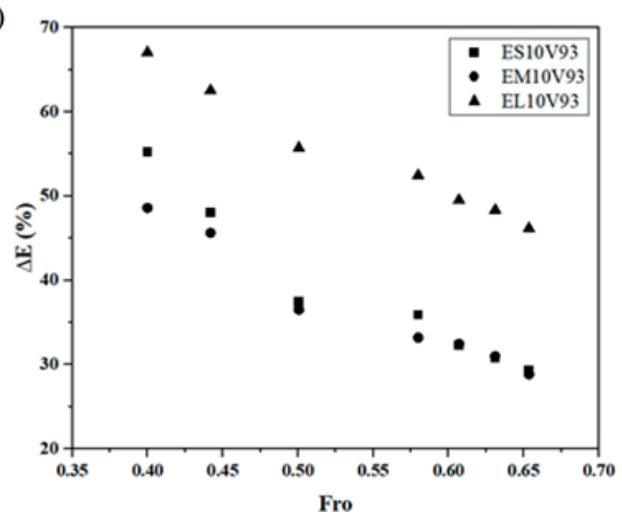

(d)

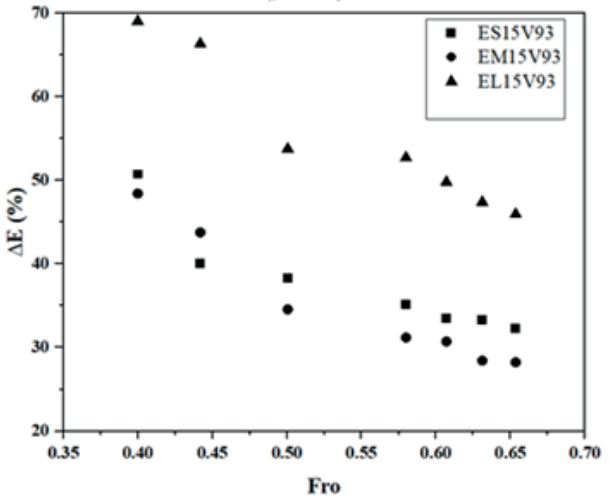

(f)

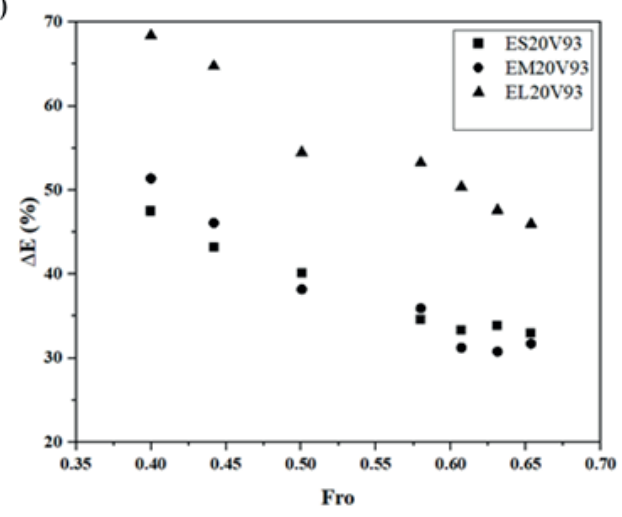

Fig. 6 Relative energy loss: By keeping the Distance $\left(L_{d v}\right)$ and porosity $\left(P_{R}\right)$ constant with the type of embankment varied, (a) ES10V97, EM10V97, EL10V97, (b) ES10V93, EM10V93, EL10V93, (c) ES15V97, EM1V97, EL15V97, (d) ES15V93, EM15V93, EL15V93 (e) ES20V97, EM20V97, 


\subsubsection{Constant type of embankment and distance $\left(L_{d v}\right)$} against varying porosity $\left(P_{R}\right)$

The effect of porosity or density of vegetation on energy dissipation is analyzed by keeping the size of embankment and $L_{d v}$ constant and porosity is varying. Thus decreasing the porosity from $97 \%$ to $93 \%$, the energy dissipation increases. The same trend of decreasing energy dissipation with increasing $F r_{o}$ is observed for all the cases presented in Fig. 7(a-c). The change in energy dissipation is more prominent in the cases having large embankment combination than small and medium embankment combinations, as the difference in means $\left(\mu_{1}-\mu_{2}\right)$ is greater than $L S_{c r}$ for the cases having large embankment, as shown in Table 2 (Appendix A). The results show that there are only two groups i.e., EL15V97-EL15V93 and EL20V97-EL20V93 are showing significant differences (SD), as presented in Table 2 (Appendix A). The maximum increase in energy dissipation due to vegetation density decrease is $18 \%$, between EL15V97 and EL15V93. The energy loss increases with vegetation porosity because of increased friction between the flow and the vegetation elements and its canopy as well as increased turbulent kinetic energy generation.

(a)

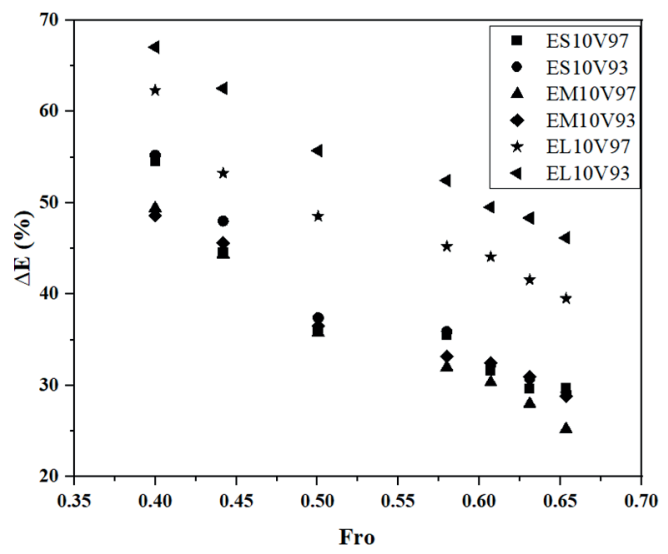

\subsubsection{Constant type of embankment and porosity} against varying distance $\left(L_{d v}\right)$

The effect of distance $L_{d v}$ on the flood energy dissipation is not as significant as the other factors like vegetation density and embankment size. This is verified by the LS method in which the mean difference $\left(\mu_{1}-\mu_{2}\right)$ for all pairs presented in Table 2 (Appendix A) is less than $L S_{c r}$ implying that no significant difference between the mean values is observed. The maximum increase observed is $7.5 \%$ between EM15V93 to EM20V93. The same trend of decreasing energy dissipation with increasing Fro is observed for all the cases presented in Fig. 8(a-f).

\section{Discussion}

The damages caused by different natural events like floods and tsunamis have turned the attention of researchers towards the combination of hard solutions (embankments, sea walls, tsunami gates etc.) and soft solutions (vegetation, coral reefs etc.). For single defense system, damage to trees and houses happens due to high energy of flow [28]. On the other hand, the regions where only embankment is used as a defense structure, the overtopping water overwhelm the

(b)

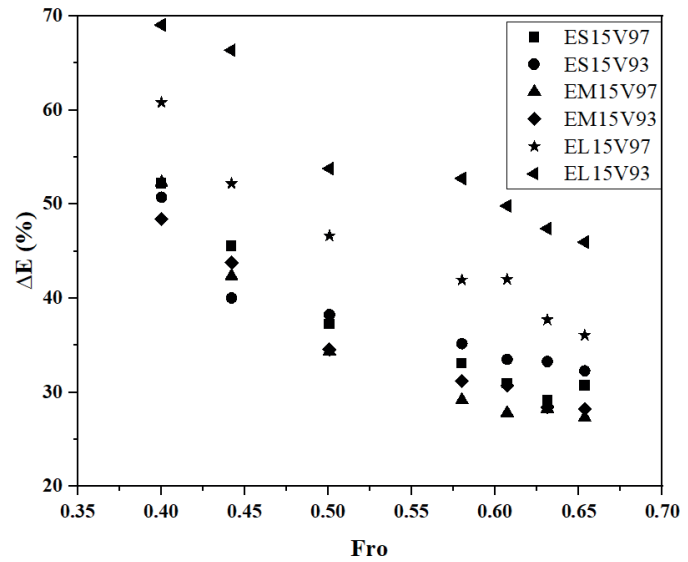

(c)

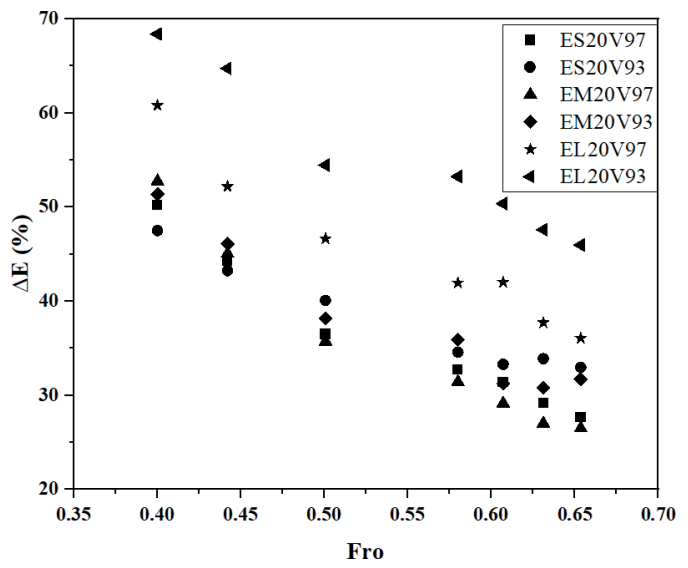

Fig. 7 Relative Energy loss by keeping the type of embankment and $L_{d v}$ constant with varying $P_{R}$; ES10V97, ES10V93, EM10V97, EM10V93, EL10V97, EL10V93; (b) ES15V97, ES15V93, EM15V97, EM15V93, EL15V97, EL15V93 and (c) ES20V97, ES20V93, EM20V97, EM20V93, EL20V97, 


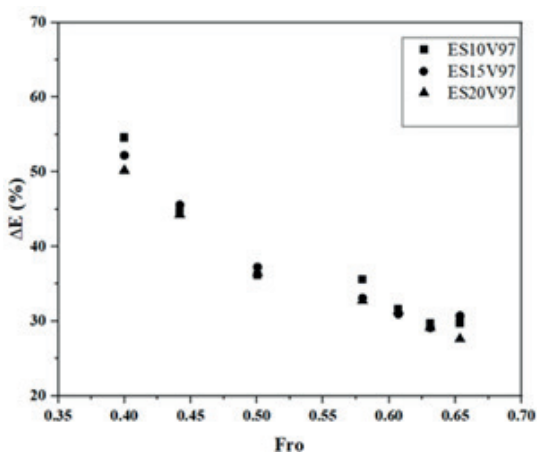

(a)

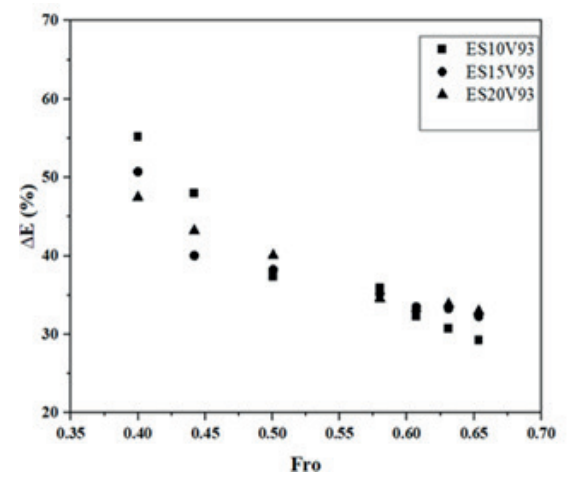

(d)

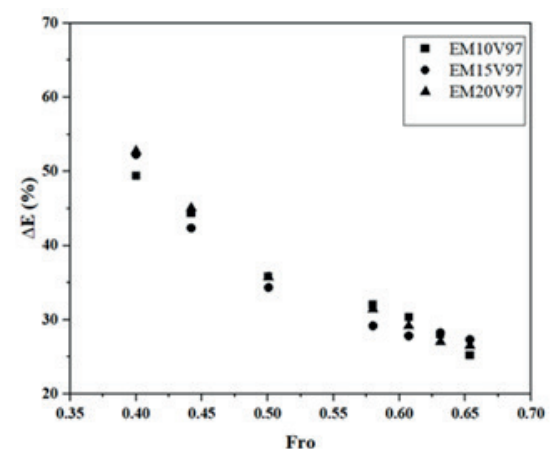

(b)

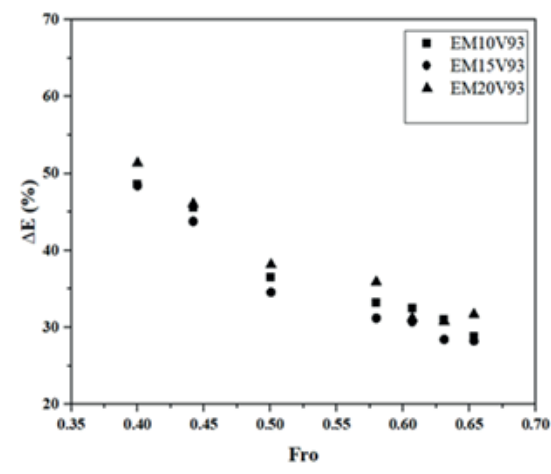

(e)

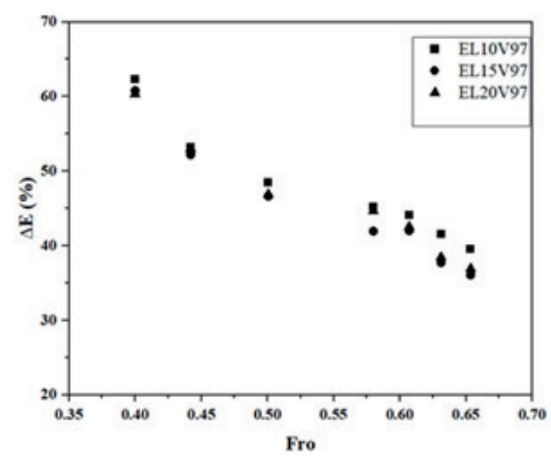

(c)

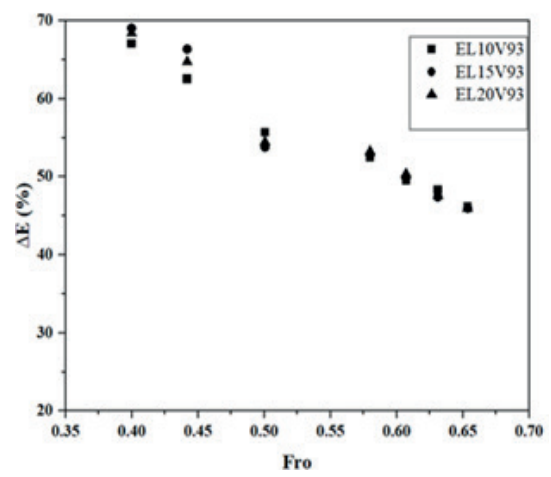

(f)

Fig. 8 Relative Energy loss by keeping the type of embankment and $P_{R}$ constant with $L_{d v}$ varied; (a) ES10V97, ES15V97, ES20V97; (b) ES10V93, ES15V93, ES20V93; (c) EM10V97, EM15V97, EM20V97; (d) EM10V93, EM15V93, EM20V93; (e) EL10V97, EL15V97, EL20V97,; (f) EL10V93, EL15V93, EL20V93

nearby structures and the human properties [29]. The flow velocity reduces when flow passes through a composite defense system due to the contraction of flow through vegetation and the formation of expansion region on the downstream of vegetation resulting in the dissipation of flow energy. Previously, composite defense structures in the form of embankment and vegetation are tested under supercritical flow conditions. Moreover, the flow regime is also examined, and the formation of hydraulic jump is categorized [2]. A hydraulic jump is used as an energy dissipator in single defense system comprising of vegetation [17] or some hydraulic structures like a chute, dam spillway, or embankment dam [30].

The current study investigates the flood energy dissipation phenomenon through composite defense system of embankment followed by emergent vegetation under subcritical flow. The idea of composite defense system has been generated by the damages caused by Tsunami (2011) in Japan [2]. The effects of Tsunami waves were tested with various experimental options, and it has reported in the literature that the Tsunami flow is either subcritical or critical [32]. In the current study, the hydraulic jump is classified into two types depending upon the location of formation of hydraulic jump either on the bed of channel (jump A) or at the downstream glacis of embankment (jump B). These major types are further subdivided into types A1, A2, B1 and B2. The results show that for small embankment cases (ES10V97, ES15V97, ES20V97, ES10V93, ES15V93 and ES20V93), type A is observed for almost $78.7 \%$ cases and type B is observed for $21.3 \%$ cases. By increasing the size of embankment from small to medium, the percentage of type A jump decreases and consequently type B increases. For all medium size embankment cases (EM10V97, EM15V97, EM20V97, EM10V93, EM15V93 and EM20V93), type A is observed for almost $59.5 \%$ cases and type B is observed for $41.5 \%$ cases. By further increasing the size of embankment from medium to large only type B is observed for all the cases. The density of vegetation also effects the type of hydraulic jump. The denser vegetation offers more resistance hence more water accumulates on the upstream of vegetation resulting in the formation of type A2 and B2 in which the depth of water is higher on the upstream of vegetation than on the downstream and inside the vegetation. For type A, water surface fluctuations are observed within Ldv, however, these fluctuations are reduced when hydraulic jump shifted 
towards the slope of the embankment i.e., type B jump same as observed by researcher in the past [2]. The safety of hydraulic defense structure depends upon the location of the hydraulic jump, and it is considered safest if its position is at the embankment toe with the subcritical regime of the jump downstream the embankment's toe [14]. Ultimately, the force on the vegetation patch decreases. Hence, type B jump is safest for the defense system and the surface erosion will also be reduces due to type B jump. However, the safety of erodible embankment will be reduced. The effect of type or height of embankment, distance between the embankment and vegetation and the density of vegetation on the type of hydraulic jump is analyzed in detail. Against the lower values of Froude numbers, the hydraulic jump formed at $L_{d v}$ while it moved towards the embankment for higher values. Similar phenomena is observed previously [31]. In this study, type B jump is observed for all the cases of large embankment in combination with vegetation of porosity $93 \%$. Hence, Type A jump is recommended for the cases where embankment is erodible, and the chances of tree breaking is less. On the other hand, Type B jump is recommended for the cases where embankment is non-erodible because in Type B jump hydraulic jump originates on the downstream glacis of embankment.

In this study, it is found that important variations in flood energy dissipation are observed by changing the size of embankment from small or medium to large embankment, as also verified by applying the LS method for various cases. The energy dissipation variation is maximum for large embankment cases. The maximum increase in energy dissipation is $18 \%$ due to decreasing vegetation porosity (from $97 \%$ to $93 \%$ ) and the maximum energy dissipation is in between $46 \%$ and $69 \%$ in the case of large embankments (EL15V93). The role of double embankments has been analyzed previously and results showed that the vegetation on embankment and spacing between the two embankments (i.e., $0 \mathrm{~cm}, 5 \mathrm{~cm}, 10 \mathrm{~cm}$, and $15 \mathrm{~cm}$ ) are increasing the energy dissipation [9]. In this study the selected spacings between the embankment and vegetation zone are $10 \mathrm{~cm}, 15 \mathrm{~cm}$ and $20 \mathrm{~cm}$. The results show that the effect of selected spacings between the embankment and vegetation is least among the other two factors i.e., embankment size and vegetation porosity, which is also verified by applying the LS method. However, the spac$\operatorname{ing} L_{d v}$ will affect the energy dissipation if a broader range of distances will be selected. The effect of spacing can be analyzed in the future by considering larger spacings between the embankment and vegetation. The maximum increase of energy dissipation due to increase of spacing is $7.5 \%$. The composite defense system comprising of embankment of higher size and vegetation of lower porosity will be highly effective against the safety of the defense structure and the relative energy dissipation.

\section{Conclusions}

In light of the results obtained in this experimental study, the following conclusions can be summarized.

a. The hydraulic jump within the composite defense system, comprising of embankment and vegetation, is observed and categorized in two types based on the initiation point of hydraulic jump. The jump formed on the bed of channel i.e., within $L_{d v}$ is termed as type A and the jump formed on the downstream glacis of embankment model is called type B. For small embankment cases, type $\mathrm{A}$ is observed for almost $78.7 \%$ cases and type $\mathrm{B}$ is observed for $21.3 \%$ cases. By increasing the size of embankment from small to medium, the percentage of type A jump decreases and consequently type B increases. For all medium embankment cases (EM10V97, EM15V97, EM20V97, EM10V93, EM15V93 and EM20V93), type A is observed for almost $59.5 \%$ cases and type B is observed for $41.5 \%$ cases. By further increasing the size of embankment from medium to large only type B for all the cases.

b. The density of vegetation also affects the type of hydraulic jump. The denser vegetation offers more resistance hence more water accumulates on the upstream of vegetation resulting in the formation of type A2 and B2 in which the depth of water is higher on the upstream of vegetation than on the downstream and inside vegetation.

c. The maximum energy dissipation range is $46-69 \%$ and the maximum average energy dissipation is $55 \%$ for the EL15V93 case.

d. The maximum increment of energy dissipation is $18 \%$ when porosity is decreased from $97 \%$ to $93 \%$ and the maximum increment in energy dissipation is $7.5 \%$ when the spacing increased between the embankment and vegetation from $10 \mathrm{~cm}$ to $20 \mathrm{~cm}$. Therefore, the size of embankment and the porosity of vegetation influence energy dissipation more than the spacing between embankment and vegetation zone.

e. Keeping in view the results Type A jump is recommended for the cases where embankment is erodible, and the chances of tree breaking is less. On the other hand, Type B jump is recommended for the cases where embankment is non-erodible because in Type B jump hydraulic jump originates on the downstream glacis of embankment. 
In the current study, the range of spacing $L_{d v}$ is limited that is why its effect is not prominent. However, the effect of spacing $L_{d v}$ can be examined by testing a broader range within the composite defense system.

\section{References}

[1] Ali, S., Uijttewaal, W. S. J. "Flow resistance of vegetated oblique weir-like obstacles during high water stages", Hydrology and Earth System Sciences, 18(1), pp. 1-14, 2014.

https://doi.org/10.5194/hess-18-1-2014

[2] Muhammad, R. A. H., Tanaka, N. "Energy Reduction of a Tsunami Current through a Hybrid Defense System Comprising a Sea Embankment Followed by a Coastal Forest", Geosciences, 9(6), Article number: 247, 2019.

https://doi.org/10.3390/geosciences9060247

[3] Zaha, T., Tanaka, N., Kimiwada, Y. "Flume experiments on optimal arrangement of hybrid defense system comprising an embankment, moat, and emergent vegetation to mitigate inundating tsunami current", Ocean Engineering, 173, pp. 45-57, 2019. https://doi.org/10.1016/j.oceaneng.2018.12.054

[4] Rashedunnabi, A. H. M., Tanaka, N. "Effectiveness of double-layer rigid vegetation in reducing the velocity and fluid force of a tsunami inundation behind the vegetation", Ocean Engineering, 201, Article number: 107142,2020

https://doi.org/10.1016/j.oceaneng.2020.107142

[5] Pasha, G. A., Tanaka, N., Yagisawa, J., Achmad, F. N. "Tsunami mitigation by combination of coastal vegetation and a backward-facing step", Coastal Engineering Journal , 60(1), pp. 104-125, 2018. https://oi.org/10.1080/21664250.2018.1437014

[6] Tanaka, N., Yasuda, S., Iimura, K., Yagisawa, J. "Combined effects of coastal forest and sea embankment on reducing the washout region of houses in the Great East Japan tsunami", Journal of Hydroenvironment Research, 8, pp. 270-280, 2014.

https://doi.org/10.1016/j.jher.2013.10.001

[7] Ahmed, A., Ghumman, A. R. "Experimental Investigation of Flood Energy Dissipation by Single and Hybrid Defense System", Water, 11(10), Article number: 1971, 2019.

https://oi.org/10.3390/w11101971

[8] Tanaka, N., Igarashi, Y. "Multiple defense for tsunami inundation by two embankment system and prevention of oscillation by trees on embankment", presented at 20th Congress of IAHR APD Congress, Colombo, Sri Lanka, Aug. 28-31, 2016.

[9] Temmerman, S., Meire, P., Bouma, T. J., Herman, P. M. J., Ysebaert, T., De Vriend, H. J. "Ecosystem-based coastal defence in the face of global change", Nature, 504, pp. 79-83, 2013. https://doi.org/10.1038/nature12859

[10] Tokida, K., Tanimoto, R. "Lessons for countermeasures using earth structures against tsunami obtained in the 2011 off the Pacific Coast of Tohoku Earthquake", Soils and Foundations, 54(4), pp. 523-543, 2014.

https://doi.org/10.1016/j.sandf.2014.07.001

\section{Acknowledgements}

This study was funded by University of Engineering \& Technology, Taxila, Pakistan. The authors acknowledge Ghufran Ahmed Pasha for his useful comments and great help during the whole of this research.

[11] Usman, F., Murakami, K., Basuki, E. "Study on Reducing Tsunami Inundation Energy by the Modification of Topography Based on Local Wisdom", Procedia Environmental Sciences, 20, pp. 642650,2014 https://doi.org/10.1016/j.proenv.2014.03.077

[12] Matsuyama, M., Uchino, D., Hashi, K., Tanaka, Y., Sakakiyama, T., Nakamura, J., Inaba, D. "Experimental Study on the Effect of a Barrier against Tsunami Flowing over a Mound", Journal of Japan Society of Civil Engineering, B (Coastal Engineering), 68(2), pp. 236-240, 2012. https://doi.org/10.2208/kaigan.68.i_236

[13] Chow, V. T "Open-channel hydraulics", McGraw-Hill, New York, NY, USA, 1959.

[14] Kawagoshi, N., Hager, W. H. "B-jump in sloping channel, II", Journal of Hydraulic Research, 28(4), pp. 461-480, 1990. https://doi.org/10.1080/00221689009499060

[15] Black, A. "Restoration: Bridging the Gaps A Graphic Translation of Ecological Restoration", MSc Thesis, University of Massachusetts, 2016.

[16] Pasha, G. A., Tanaka, N. "Undular hydraulic jump formation and energy loss in a flow through emergent vegetation of varying thickness and density", Ocean Engineering, 141, pp. 308-325, 2017. https://doi.org/10.1016/j.oceaneng.2017.06.049

[17] Castro-Orgaz, O., Chanson, H. "Minimum Specific Energy and Transcritical Flow in Unsteady Open-Channel Flow", Journal of Irrigation and Drainage Engineering, 142(1), Article number: 04015030, 2016.

[18] Al-hashimi, S. A. M., Madhloom, H. M.,Nahi, T. N., Al-ansari, N. "Channel Slope Effect on Energy Dissipation of Flow over Broad Crested Weirs", Engineering, 8(12), pp. 837-851, 2016. https://doi.org/10.4236/eng.2016.812076

[19] Pavlin, Ž., Kuspilić, N. "Reference water levels for the design of dykes and earthfill dams", GRAĐEVINAR, 70(3), pp. 225-233, 2018 .

https://doi.org/10.14256/jce.2093.2017

[20] Mahtabi, G., Arvanaghi, H. "Experimental and numerical analysis of flow over a rectangular full-width sharp-crested weir", Water Science and Engineering, 11(1), pp. 75-80, 2018. https://doi.org/10.1016/j.wse.2018.03.004

[21] Usman, F., Murakami, K. "Study on reducing tsunami inundation energy by the modification of topography based on local wisdom", Journal of Japan Society of Civil Engineering, B (Ocean Engineering), 68(2), pp. 66-71, 2012.

[22] Tanvir, M. A., Siddiqui, M. T., Shah, A. H. "Growth and Price Trend of Eucalyptus camaldulensis in Central Punjab", International Journal of Agriculture and Biology, 4(3), 344-346, 2002. 
[23] Huai, W. X., Zeng, Y. H., Xu, Z. G., Yang, Z. H. "Three-layer model for vertical velocity distribution in open channel flow with submerged rigid vegetation", Advances in Water Resources, 32(4), pp. 487-492, 2009.

https://doi.org/10.1016/j.advwatres.2008.11.014

[24] Huai, W., Wang, W., Hu, Y., Zeng, Y., Yang, Y. "Analytical model of the mean velocity distribution in an open channel with double-layered rigid vegetation", Advances in Water Resources, 69, pp. 106113, 2014.

https://doi.org/10.1016/j.advwatres.2014.04.001

[25] Chanson, H. "Current knowledge in hydraulic jumps and related phenomena. A survey of experimental results", European Journal of Mechanics B/Fluids, 28(2), pp. 191-210, 2009.

https://doi.org/10.1016/j.euromechflu.2008.06.004
[26] Lee, S., Lee, D. K. "What is the proper way to apply the multiple comparison test?", Korean Journal of Anesthesiology, 71(5), pp. 353-360, 2018.

https://doi.org/10.4097/kja.d.18.00242

[27] Darcy, H. P. G., Bazin, H. "Recherches hydrauliques, Imprimerie Impériales (Hydraulic research)", Imprimerie Impériales, Paris, France, 1865. (in French)

[28] Hager, W. H. "B-jump in sloping channel", Journal of Hydraulic Research, 26(5), pp. 539-558, 1988.

https://oi.org/10.1080/00221688809499192

[29] Pasha, G. A., Tanaka, N. "Characteristics of a Hydraulic Jump Formed on Upstream Vegetation of Varying Density and Thickness", Journal of Earthquake and Tsunami, 14(3), Article number: 2050012, 2020. https://doi.org/10.1142/S1793431120500128 


\section{Appendix-A}

Table 2 Comparison of various cases of composite defense system against energy dissipation

\begin{tabular}{|c|c|c|c|c|c|}
\hline Case & & & $\mu_{1}-\mu_{2}$ & $L S_{c r}$ & Remarks \\
\hline \multirow{18}{*}{ 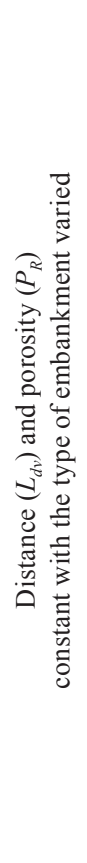 } & ES10V97 & EM10V97 & 2.4 & 8.9 & NSD \\
\hline & ES10V97 & EL10V97 & 10.4 & 8.9 & SD \\
\hline & EM10V97 & EL10V97 & 12.8 & 8.9 & SD \\
\hline & ES10V93 & EM10V93 & 1.8 & 9.4 & NSD \\
\hline & ES10V93 & EL10V93 & 16.1 & 9.4 & SD \\
\hline & EM10V93 & EL10V93 & 18.0 & 9.4 & SD \\
\hline & ES15V97 & EM15V97 & -2.5 & 10.1 & NSD \\
\hline & ES15V97 & EL15V97 & 8.3 & 10.1 & NSD \\
\hline & EM15V97 & EL15V97 & 10.8 & 10.1 & SD \\
\hline & ES15V93 & EM15V93 & 2.6 & 8.9 & NSD \\
\hline & ES15V93 & EL15V93 & 17.4 & 8.9 & SD \\
\hline & EM15V93 & EL15V93 & 20.0 & 8.9 & SD \\
\hline & ES20V97 & EM20V97 & -0.6 & 10.0 & NSD \\
\hline & ES20V97 & EL20V97 & 10.1 & 10.0 & SD \\
\hline & EM20V97 & EL20V97 & 10.7 & 10.0 & SD \\
\hline & ES20V93 & EM20V93 & 0.0 & 7.7 & NSD \\
\hline & ES20V93 & EL20V93 & 17.0 & 7.7 & SD \\
\hline & EM20V93 & EL20V93 & 17.1 & 7.7 & SD \\
\hline \multirow{9}{*}{ 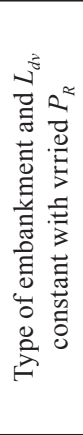 } & ES10V97 & ES10V93 & 1.0 & 9.2 & NSD \\
\hline & EM10V97 & EM10V93 & 1.6 & 9.2 & NSD \\
\hline & EL10V97 & EL10V93 & 6.8 & 9.2 & NSD \\
\hline & ES15V97 & ES15V93 & 0.6 & 9.2 & NSD \\
\hline & EM15V97 & EM15V93 & 0.5 & 9.2 & NSD \\
\hline & EL15V97 & EL15V93 & 9.7 & 9.2 & SD \\
\hline & ES20V97 & ES20V93 & 1.9 & 9.2 & NSD \\
\hline & EM20V97 & EM20V93 & 2.5 & 9.2 & NSD \\
\hline & EL20V97 & EL20V93 & 9.6 & 9.2 & SD \\
\hline \multirow{18}{*}{ 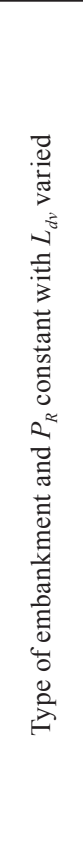 } & ES10V97 & ES15V97 & 0.4 & 8.4 & NSD \\
\hline & ES10V97 & ES20V97 & 1.4 & 8.4 & NSD \\
\hline & ES15V97 & ES20V97 & 1.0 & 8.4 & NSD \\
\hline & EM10V97 & EM15V97 & 0.5 & 10.6 & NSD \\
\hline & EM10V97 & EM20V97 & -0.4 & 10.6 & NSD \\
\hline & EM15V97 & EM20V97 & -0.9 & 10.6 & NSD \\
\hline & EL10V97 & EL15V97 & 2.4 & 9.3 & NSD \\
\hline & EL10V97 & EL20V97 & 1.7 & 9.3 & NSD \\
\hline & EL15V97 & EL20V97 & -0.8 & 9.3 & NSD \\
\hline & ES10V93 & ES15V93 & -0.8 & 8.4 & NSD \\
\hline & ES10V93 & ES20V93 & -0.5 & 8.4 & NSD \\
\hline & ES15V93 & ES20V93 & 0.3 & 8.4 & NSD \\
\hline & EM10V93 & EM15V93 & 1.5 & 9.0 & NSD \\
\hline & EM10V93 & EM20V93 & -1.3 & 9.0 & NSD \\
\hline & EM15V93 & EM20V93 & -2.8 & 9.0 & NSD \\
\hline & EL10V93 & EL15V93 & -0.5 & 9.5 & NSD \\
\hline & EL10V93 & EL20V93 & -0.4 & 9.5 & NSD \\
\hline & EL15V93 & EL20V93 & 0.1 & 9.5 & NSD \\
\hline
\end{tabular}

*NSD = No Significant Difference; *SD = Significant Difference 